\title{
Understanding Pd Hydrogenation Catalysts: When Nature of the Reactive Molecule Controls the Nature of the Catalyst Active Phase
}

("dedicated to the $50^{\text {th }}$ anniversary of the Catalysis Society of Japan")

\author{
D. Teschner* ${ }^{1}$, Z. Révay ${ }^{2}$, J. Borsodi $^{2}$, M. Hävecker ${ }^{1}$, A. Knop-Gericke ${ }^{1}$, R. Schlögl ${ }^{1}$, D. Milroy ${ }^{3}$, \\ S. D. Jackson ${ }^{3}$, D. Torres ${ }^{4}$, P. Sautet ${ }^{4}$
}

\author{
${ }^{1}$ Fritz-Haber-Institut der Max-Planck-Gesellschaft, Faradayweg 4-6, D-14195 Berlin, Germany, \\ ${ }^{2}$ Institute of Isotopes, Hungarian Academy of Sciences, Post Office Box 77, Budapest H-1525, Hungary \\ ${ }^{3}$ WestCHEM, Department of Chemistry, University of Glasgow, Glasgow G128 QQ, Scotland, UK \\ ${ }^{4}$ Université de Lyon, Institut de Chimie, Laboratoire de Chimie, Ecole Normale Supérieure de Lyon and CNRS \\ 46 Allée d'Italie, 69364 Lyon, Cedex 07 (France)
}

${ }^{*}$ Corresponding author: e-mail teschner@ffhi-berlin.mpg.de,

Received: 7 May 2008

Keywords: density functional calculation, hydrogenation, in situ XPS, Pd, PGAA

\begin{abstract}
Catalytic hydrogenations are one of the most important processes of the chemical industry and selectivity is a timely issue. The presence of carbon-carbon triple bond compounds in the alkene stream is undesirable in both chemical and polymer-grade propylene and ethylene, due to poisoning of the polymerization catalysts. ${ }^{[1]}$ Starting with a mixture of alkynes and alkenes, Pd-based catalysts are known to be capable to selectively hydrogenate the triple bonds leaving the olefinic function intact. The presence of carbonaceous deposits was assumed to play a key role, influencing the performance of the catalyst. ${ }^{[2]}$ Although the origin of this behavior has been a puzzle so far, very recently a relation between selectivity and subsurface chemistry has been established, showing that the population of subsurface sites of Pd, by either hydrogen or carbon, governs selective alkyne hydrogenation. ${ }^{[3]}$ Hydrogen in the subsurface has been demonstrated to play a crucial role by hydrogenating surface species effectively. ${ }^{[4,5]}$

Herein, we extend our previous studies addressing the fundamental differences of carbon-carbon double or triple bond hydrogenation over Pd. The present results provide solid evidence that the difference in the hydrogenation of alkynes/alkenes stems back to the strongly different state of the near-surface region. We show that the formation of the PdC surface phase is directly related to the nature of the
\end{abstract}

reactive molecule, being a general pattern for alkyne hydrogenation and absent for alkenes. We show in addition that the presence of this PdC phase affects the surface chemistry by decreasing the amount of more active subsurface hydrogen with respect to the more selective onsurface ones, hence controlling the state of the Pd catalyst surface under reactive conditions.

In order to unravel the nature of the active Pd surface, we have studied, by in situ X-ray Photoelectron Spectroscopy (XPS), the near-surface region of palladium under various hydrogenation conditions for a set of reacting alkynes and alkenes. Under the reduced pressure conditions of 1 mbar, alkynes are hydrogenated to alkenes while alkene feeds are transformed to alkanes, just as at more realistic conditions. Figure 1 summarizes the state of palladium under such hydrogenation conditions. The palladium $3 \mathrm{~d}$ core level was found to be the best indicator of the nearsurface state. ${ }^{[3]}$ While the metallic bulk peak is observed at $335 \mathrm{eV}$, a strong new component (filled peak) appears when alkynes are hydrogenated selectively. This peak at $335.6 \mathrm{eV}$, when dominating the spectrum, is characteristic of the carbon modified, PdC surface phase. Note that adsorbates give rise to a surface state at similar energy (adsorbate induced surface core level shift); however at the 

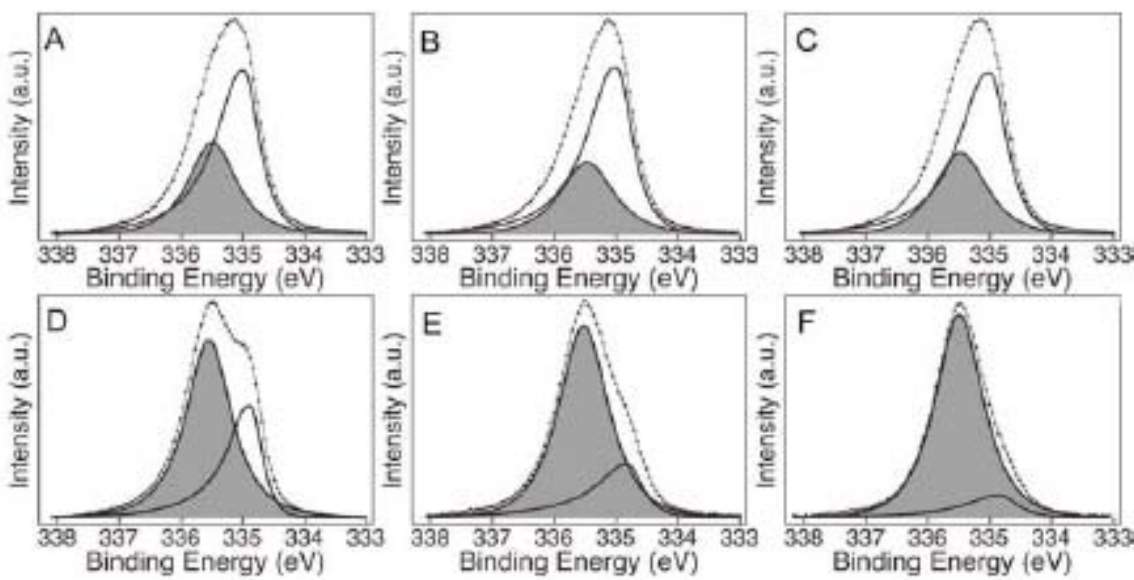

Fig.1: Comparison of in situ $\mathrm{Pd} 3 \mathrm{~d}_{5 / 2}$ spectra of Pd foil under alkene and alkyne hydrogenation at $1 \mathrm{mbar}\left(\mathrm{H}_{2} / \mathrm{C}_{\mathrm{x}} \mathrm{H}_{\mathrm{y}}: 9 / 1\right)$ and $343-353 \mathrm{~K}$. A: 1pentene; B: propene; C: ethylene; D: 1-pentyne; E: propyne; F: acetylene.

applied $720 \mathrm{eV}$ excitation energy the contribution from this state should be less than $25 \%$ of the whole peak intensity.

Clearly, all the investigated alkynes induce the formation of $\mathrm{PdC}{ }^{[3]}$ since the higher binding energy component dominates the spectra. The only difference seems to be in the amount of carbon dissolved in the Pd lattice: for lower chain alkynes more carbon is dissolved in Pd making the $\mathrm{PdC}$ phase thicker. In contrast, hydrogenating alkenes generates a much weaker signal at the higher binding energy side of bulk Pd. According to our fitting procedure, this peak corresponds roughly $1 / 3$ of the whole signal intensity, which indicates that some carbon is indeed dissolved in the upper part of the metal lattice. However, we attribute the lack of a clearly distinguishable $335.6 \mathrm{eV}$ component to the absence of PdC. These results show the widely different nature of the surface under alkyne or alkene feed.

Since industrially palladium is used to hydrogenate alkynes from a mixed alkyne/alkene feed, we have investigated the temporal evolution of the surface using timeresolved in situ XPS, when co-dosing propyne to a propene $/ \mathrm{H}_{2}$ feed. As expected from the previous results, the $\mathrm{Pd}$ $3 \mathrm{~d}$ core level was dominated by the bulk component in propene $+\mathrm{H}_{2}$ feed (see Figure $\mathrm{S} 1$ in the Supporting Information). It was however strongly attenuated in the first few minutes after propyne was introduced, and the proportion of the higher binding energy component slowly increased further in the mixed feed (Fig. 2A). After half an hour timeon-stream the ratio of the two Pd components was inverted, with $\mathrm{PdC}$ reaching $2 / 3$ of the whole signal intensity. The observed temporal profile indicates that the carbon uptake can be divided into two processes: an initial fast step and a second slower step. Since carbon energetically prefers to occupy the first subsurface octahedral sites (vide infra), we assign the fast step to the occupation of these sites. The very first propyne (alkyne) molecules chemisorbing on Pd undergo fragmentation and the fragments, likely individual carbon atoms, penetrate the surface. Since the second step of $\mathrm{PdC}$ formation is much slower, the build up of additional mixed Pd-C layers should be energetically less favorable. The calculated ${ }^{[6]}$ growth rate of $\mathrm{PdC}$ is $\sim 7$ Angstöm/hours,
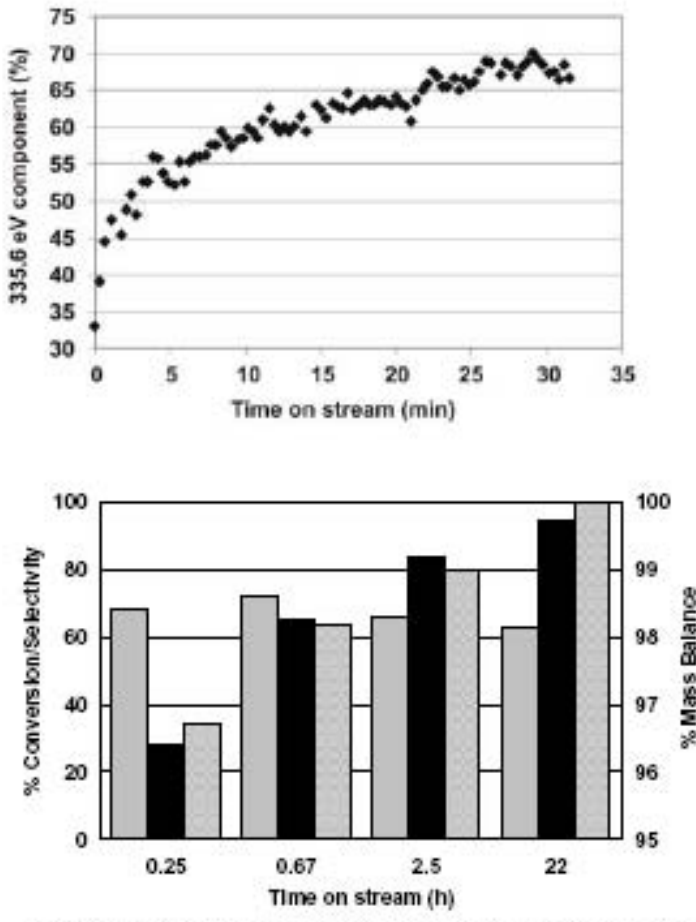

$\square$ Acetylene Conversion $\square$ Ethylene Selectivity $\square$ Mass Balance

Fig. 2: (A) Temporal evolution of the proportion of the higher binding energy $\mathrm{Pd} 3 \mathrm{~d}_{5 / 2}$ component at $\sim 335.6 \mathrm{eV}$ after adding 0.1 mbar propyne in the feed of $0.9 \mathrm{mbar}^{\mathrm{H}_{2}}$ and 0.1 mbar propene according to time-resolved in situ XPS. T: $343 \mathrm{~K}$. (B) Change in selectivity of acetylene hydrogenation at 20 bar and $323 \mathrm{~K}$. Catalyst Pd/alumina, GHSV: 5000, hydrogen:ethylene:acetylene ratio, $25: 25: 1$.

which is equivalent to a diffusion coefficient of carbon $\left(\mathrm{D}_{\mathrm{c}}\right)$ of $\sim 10^{-22} \mathrm{~m}^{2} / \mathrm{s}$, in good agreement with the results of Yokoyama et al. ${ }^{[7]}\left(10^{-23} \mathrm{~m}^{2} / \mathrm{s}\right)$ extrapolated to our temperature. 
Similar behavior in carbon uptake is also seen over a Pd catalyst under industrially relevant conditions. Figure $2 \mathrm{~B}$ shows the conversion and selectivity obtained from acetylene hydrogenation. Over the fresh catalyst there is poor alkene selectivity but with time this increases from 27 $\%$ to $95 \%$. Over the same period the mass balance increases from 96.5 to $100 \%$ showing that as carbon is deposited the selectivity increases. Note that there is little change in activity.

To hydrogenate a given chemical functionality the catalyst obviously needs hydrogen. For palladium its propensity to form subsurface or bulk dissolved hydrogen (e.g. $\beta$ hydride) makes the assignment of the active hydrogen nontrivial. Both, experimental and theoretical studies clearly indicated that hydrogen, when emerging from the subsurface/bulk to the surface, could very effectively hydrogenate different adsorbates. ${ }^{[4,5]}$ Provided subsurface hydrogen plays an important role in our processes, the occupation of the first subsurface layer with carbon could be critical in the hydrogenation path. To be able to measure and quantify hydrogen dissolved in palladium we have developed the Prompt Gamma Activation Analysis (PGAA) ${ }^{[8]}$ to an in situ technique. ${ }^{[9]}$ In the PGA process the nuclei of the chemical elements in the investigated specimen can capture neutrons, and during the de-excitation they emit characteristic prompt gamma photons, which can be detected using an appropriate spectrometer. Hydrogen has a medium neutron capture cross section, but gives a strong signal in the gamma spectrum, thus hydrogen atoms dissolved in palladium can be analyzed by PGAA with a high sensitivity.

Table 1: Conversion, product selectivities and atomic $\mathrm{H} / \mathrm{Pd}$ ratios during in situ PGAA experiments. Sample: $7 \mathrm{mg}$ Pd black; in flowing hydrogen, under 1-pentyne or 1-pentene hydrogenation (after $\mathrm{H}_{2}$ pretreatment); at 1 bar and room temperature. Gas flow during hydrogenation: $4 \mathrm{ml} / \mathrm{min} \mathrm{H}_{2}$ and $1.6 \mathrm{ml} / \mathrm{min}_{5} \mathrm{H}_{\mathrm{x}}$. 1-pentyne hy-

\begin{tabular}{|c|c|c|c|}
\hline Experiments & $\begin{array}{c}\text { Conversion } \\
(\%)\end{array}$ & $\begin{array}{c}\text { Products } \\
(\%)\end{array}$ & $\mathrm{H} / \mathrm{Pd}$ \\
\hline $\mathrm{H}_{2}^{[\mathrm{l}]}\left(1^{\mathrm{tt}}\right)$ & - & - & 0.75 \\
\hline 1-pentyne $\left(1^{\text {st }}\right)$ & $21 \%$ & $\begin{array}{c}97.7 \% 1 \text {-pentene } \\
2.3 \% \text { pentane }\end{array}$ & 0.27 \\
\hline $\mathrm{H}_{2}^{[\mathrm{a}]}\left(2^{\text {nd }}\right)$ & - & - & 0.77 \\
\hline 1-pentyne $\left(2^{\text {nd }}\right)$ & $44 \%$ & $\begin{array}{l}98.1 \% 1 \text {-pentene } \\
1.9 \% \text { pentane }\end{array}$ & 0.01 \\
\hline $\mathrm{H}_{2}\left(3^{\text {th }}\right)$ & - & - & 0.88 \\
\hline 1 -pentene $\left(3^{\text {rd }}\right)$ & $99.8 \%$ & $\begin{array}{c}98.6 \% \text { pentane } \\
1.4 \% 2 \text {-pentenes }\end{array}$ & 0.77 \\
\hline
\end{tabular}

drogenation was repeated.

Table 1 compares the hydrogen content of Pd black during 1-pentyne and 1-pentene hydrogenation. When fresh samples are introduced into flowing hydrogen, the hydrogen content matched perfectly the values from the $\mathrm{Pd} / \mathrm{H}$ phase diagram. ${ }^{[10]}$ Thereafter the H/Pd ratio decreased significantly when selective hydrogenation of 1-pentyne was carried out using a feed ratio $\left(\mathrm{H}_{2} / \mathrm{C}_{5}\right)$ of 2.5. The hydrogen content in pure hydrogen, but after 1-pentyne hydrogena-

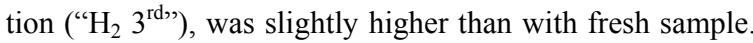
This suggests that the surface includes carbonaceous deposits containing additional hydrogen. When running 1pentene hydrogenation the hydrogen content was high, much higher than with the equivalent alkyne. This difference can be related to the XPS observations (vide infra). In order to achieve an atomistic understanding for the formation of the PdC phase we first address the incorporation of $\mathrm{C}$ into the Pd substrate. We performed self-consistent Density functional calculations (DFT) using the VASP code ${ }^{[11]}$ and a periodic $\operatorname{Pd}(111)$ model surface. (Note, the $\operatorname{Pd}(111)$ surface was used as a model aiming only to extract the main phenomena for $\mathrm{C}$ penetration and $\mathrm{PdC}$ phase formation. Hence DFT was used to qualitatively (and not quantitatively) describe the experiments.) The results of our calculations are shown in Figure 3 (top panels). We determined the average binding energy of carbon $\left(E_{b}^{C}\right)$ as a function of total $\mathrm{C}$ coverage $\left(\Theta_{C}\right)$ with different distributions of $\mathrm{C}$ between surface, subsurface and second interlayer. After exploring all possible adsorption site combinations, the most stable situation is shown.

The initial stages in the formation of the $\mathrm{PdC}$ phase proceeds via $\mathrm{C}$ incorporation from the surface to the first interlayer. Independently of $\Theta_{C}$, structures involving $\mathrm{C}$ atoms adsorbed only in the subsurface are energetically
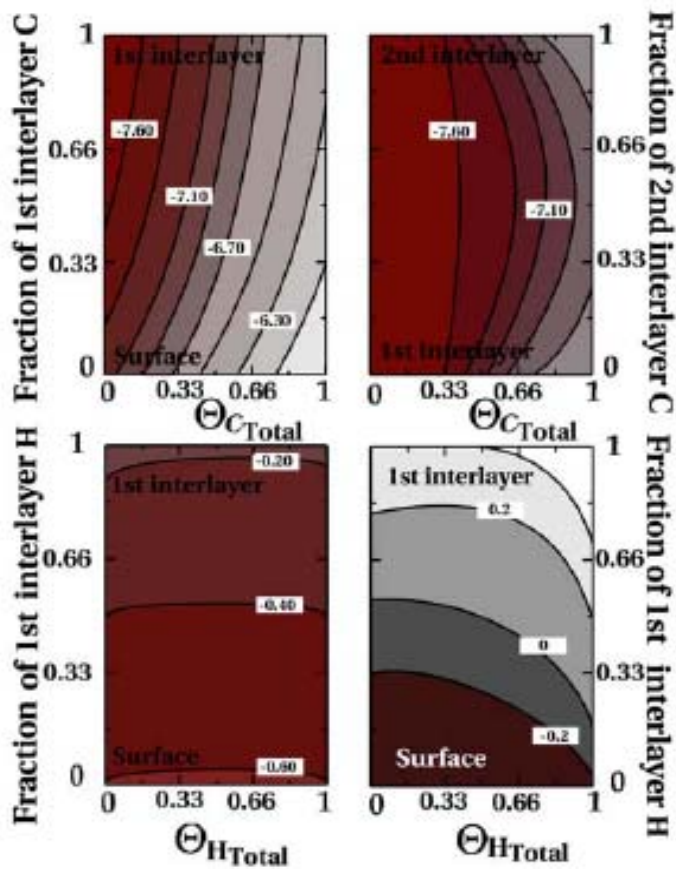

Fig. 3: (Top): Contour maps showing the average binding energy (colour coded, in $\mathrm{eV}$ ) of carbon as a function of total $\mathrm{C}$ coverage ( $\mathrm{X}$ axis) and the distribution of carbon atoms between interlayers (Y axis) using a $\mathrm{Pd}(111)$ substrate. $\mathrm{C}$ atoms are distributed between surface and $1^{\text {st }}$ interlayer sites (left panel) or between $1^{\text {st }}$ and $2^{\text {nd }}$ interlayer (right panel). (Bottom): Contour maps showing the average binding energy (colour coded, in eV) of hydrogen as a function of total $\mathrm{H}$ coverage (X axis) on $\mathrm{Pd}(111)$ (left) or on the model PdC phase (right). $\mathrm{H}$ atoms are distributed between surface and $1^{\text {st }}$ interlayer sites. 


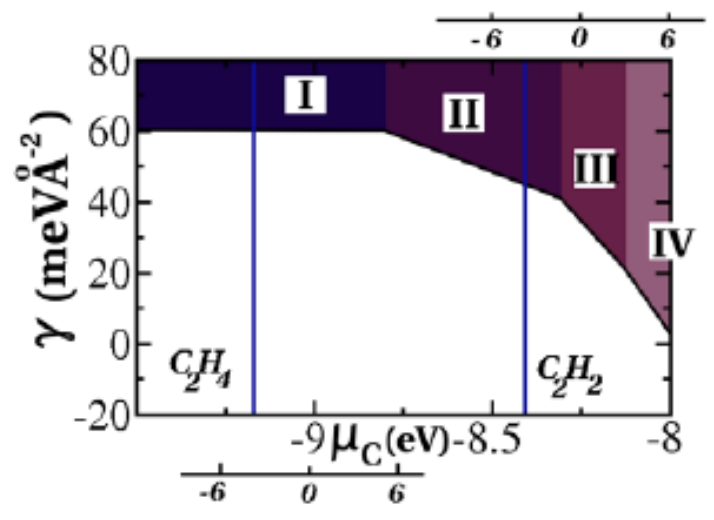

Fig. 4: Surface free energy $\gamma\left(\mathrm{meV}^{-2}\right)$ of the most stable $\operatorname{PdC}$ structures as a function of carbon chemical potential $\mu_{C}$. Structure I is the pure $\operatorname{Pd}(111)$ surface, II shows a $1 / 3$ ML C content in the first interlayer, III a 2/3 ML C content distributed among first and second interlayers, and IV a 1 ML C distributed in the first three interlayers. Vertical lines indicate the $\mu_{C}$ value for the acetylene (right line) and ethylene (left line) dissociation into $\mathrm{C}$ and $\mathrm{H}_{2}$, at pressure conditions employed in XPS $\left(10^{-4}\right.$, and $10^{-3}$ atm for $p_{C 2 H x}$ and $\left.p_{H 2}\right) . \mu_{C}$ has also been cast into a logarithmic pressure scale at a fixed temperature of $400 \mathrm{~K}$. Acetylene and ethylene pressures with respect to $p_{0}(1 \mathrm{~atm})$ are respectively shown in the bottom and top 0X axis.

favored (Figure 3 top left panel) with respect to structures involving $\mathrm{C}$ adatoms on the surface or on both the surface and subsurface. The most stable conformation corresponds to a $\sqrt{3} x \sqrt{ } 3$ distribution of $\mathrm{C}$ in the first interlayer. ${ }^{[12]} \mathrm{Re}-$ garding the $\mathrm{C}$ distribution between the first and second interlayer the situation changes. Placing the $\mathrm{C}$ atoms in the second interlayer is roughly as favorable as placing them in the first. For coverage higher than $\sim 0.3 \mathrm{ML}$, distribution of the $\mathrm{C}$ atoms on both first and second interlayer is energetically more favored than the population of a single interlayer, hence creating the thermodynamic driving force for the growth of the $\mathrm{PdC}$ phase. We considered $\mathrm{C}$ incorporation into deeper interlayers and, as a general rule, we observed a significant weakening of the average $\mathrm{C}$ binding when an increasing number of interlayers were populated; hence this will limit for deeper extension of the PdC phase.

With the aim of describing the XPS experiments presented in this paper, at constant pressures and temperature, the appropriate thermodynamic potential to consider is the Gibbs free surface energy $\gamma(T, P)$. This scheme has been successfully applied to various systems, ${ }^{[13]}$ and we will use it here to determine the thermodynamically stable composition of the surface in equilibrium with a reactive atmosphere, described by acetylene and hydrogen partial pressures ( $p_{\mathrm{C} 2 \mathrm{H} 2}$ and $p_{\mathrm{H} 2}$ respectively) at a fixed temperature $T$. Assuming that thermodynamic equilibrium applies, the environment then acts as a $\mathrm{C}$ adatoms reservoir, as it can give or take $\mathrm{C}$ atoms via the decomposition or formation of the carbon containing molecules $(\mathrm{H}$ atoms being evolved as $\mathrm{H}_{2}$ to the gas phase). The calculated free surface energy plot as a function of $\mathrm{C}$ chemical potential $\left(\mu_{C}\right)$ is shown in Figure 4. For low values of $\mu_{C}$ molecule decom- position and $\mathrm{C}$ deposition onto the surface is not favored. Only once a certain critical $\mathrm{C}$ chemical potential $\left(\mu_{C}{ }^{C r i t}\right)$ value of $-8.8 \mathrm{eV}$ is reached the $\mathrm{C}$ deposition starts.

When acetylene and hydrogen are dosed on the catalyst surface, reaction starts and the $\mathrm{C}$ chemical potential $\mu_{C}$ vary within certain well-defined boundaries. While the upper boundary $\left(\mu_{C}^{M a x}\right)$ is given by the chemical potential of carbon in the acetylene molecule, the lower boundary is given by the chemical potential of carbon in ethylene, at given $\mathrm{H}_{2}$ pressure. The formation of the PdC phase will be thermodynamically favored under reaction conditions only when $\mu_{C}$ is above the critical equilibrium $\mu_{C}{ }^{\text {Crit }}$ value for the migration of carbon to the subsurface. From Fig. 4 this is clearly the case for acetylene hydrogenation, but not for ethylene hydrogenation reaction. The stability of this phase for acetylene is dependent on the $\mathrm{H}_{2}$ pressure, because the effective chemical potential of carbon from the molecular reservoir depends on $\mathrm{H}_{2}$ pressure. At high $\mathrm{H}_{2}$ pressure, formation of the phase becomes thermodynamically inhibited.

The difference $\left(\Delta \gamma^{f}\right)$ between the surface free energy of the most stable structure and that of the $\operatorname{Pd}(111)$ surface, for the $\mu_{C}$ associated to the reactant, can be considered as a descriptor of the thermodynamic driving force for the PdC phase formation. For the hydrogenation of acetylene, at typical pressure conditions during in situ XPS $\left(10^{-4}\right.$ and $10^{-3}$ atm for $p_{\mathrm{C} 2 \mathrm{H} 2}$ and $p_{\mathrm{H} 2}$ ), and at $400 \mathrm{~K}$, the formation of the PdC phase due to the decomposition of acetylene is thermodynamically favored, with $\Delta \gamma^{f} \sim-25 \mathrm{meV} / \AA^{2}$. In contrast, the formation of the phase in the presence of ethene is disfavored, with positive $\Delta \gamma^{f}$. To get more insight into the relation of the $\mathrm{PdC}$ phase formation with respect to the nature of the reacting molecule we calculated $\Delta \gamma^{f}$ for a set of reactive molecules of the type $\mathrm{HC} \equiv \mathrm{CX}$, with $\mathrm{X}=\mathrm{CH}_{3}$, $\mathrm{H}, \mathrm{F}, \mathrm{Cl}$ (Figure S2). The formation of the PdC phase is favored for lower chain alkyne hydrogenation or when electronegative species are attached to the triple bond. This qualitatively explains the thicker PdC phase obtained experimentally when going from 1-pentyne to propyne and to acetylene.

Finally, the presence of $\mathrm{C}$ in the subsurface affects the properties of surface hydrogen. In order to analyze this influence we compared the binding energy of $\mathrm{H}$ on $\mathrm{Pd}(111)$ and on a PdC phase model. To match the experiments best, we chose structure IV of Fig 4, with the first three interlayers occupied with $1 / 3 \mathrm{C}$ coverage. The average binding energy of $\mathrm{H}\left(E^{H}{ }_{b}\right)$ as a function of total $\mathrm{H}$ coverage $\left(\Theta_{H}\right)$ was calculated with respect to the gas phase $\mathrm{H}_{2}$ molecule. On the clean $\operatorname{Pd}(111)$ surface, the binding energy does not strongly depend on the coverage (Figure 3 bottom left panel). $\mathrm{H}$ is most stable on the surface, but the penetration into the subsurface is more favored than desorption. ${ }^{[14]}$ In contrast, the bonding properties of $\mathrm{H}$ are strongly modified for $\mathrm{PdC}$ (Figure 3 bottom right panel): adsorption on the surface is weakened and, most importantly, the accumulation of $\mathrm{H}$ into the subsurface is thermodynamically disfavored. Thus one role of the PdC phase is to hinder the migration of $\mathrm{H}$ to the subsurface, hence decreasing the $\mathrm{H}^{\text {sub }} / \mathrm{H}^{\text {on }}$ ratio in the sample. The PdC phase will, in addition, prevent the migration of bulk $\mathrm{H}$ toward the surface. Clearly, DFT is in 
accord with the PGAA results, because once $\beta$-hydride has been depleted, hydrogen is hindered in replenishing the bulk, and the H/Pd ratio will be low. Hence, alkynes are hydrogenated selectively by surface hydrogen, since hydrogen cannot emerge from the bulk, if present at all. On the other hand, alkene hydrogenation occurs using subsurface hydrogen, as its concentration is high and no energetic barrier is built up by a subsurface carbon population.

All these data clearly indicate that a palladium catalyst can be considered as "living material", and its actual (near)surface state is a strong function of the experimental hydrogenation conditions. Understanding such interplays will allow designing heterogeneous catalysts to a desired reaction. The present study clearly demonstrates the significance of combining theory and experimentation in bringing understanding to a new level.

\section{Experimental Section}

In situ X-ray photoelectron spectroscopy (XPS) experiments were performed at beamlines U49/2-PGM1 and PGM2 at BESSY, Berlin. Pd 3d core levels of a Pd foil sample were recorded at normal emission with $720 \mathrm{eV}$

\section{References}

[1] a) H. Arnold, F. Dobert, J. Gaube in Hydrogenation Reaction in Handbook of Heterogeneous Catalysis, vol. 5 (Eds.: G. Ertl, H. Knozinger, J. Weitkamp) Wiley-VCH, New York, 1997, p. 2165.; b) A. Molnar, A. Sarkany, M. Varga, J. Mol. Catal. A 2001, 173, 185.

[2] A. Borodziński, G. C. Bond, Catal. Reviews 2006, 48, 91. (and references therein)

[3] a) D. Teschner, J. Borsodi, A. Wootsch, Zs. Révay, M. Hävecker, A. Knop-Gericke, S. David Jackson, R. Schlögl, Science 2008, 320, 86; b) D. Teschner, E. M. Vass, M. Hävecker, S. Zafeiratos, P. Schnörch, H. Sauer, A. Knop-Gericke, R. Schlögl, M. Chamam, A. Wootsch, A. S. Canning, J. J. Gamman, S. D. Jackson, J. McGregor, L. F. Gladden, J. Catal. 2006, 242, 16.

[4] A. D. Johnson, S. P. Daley, A. L. Utz, S. T. Ceyer, Science 1992, 257, 223.

[5] a) A. Michaelides, P. Hu, A. Alavi, J. Chem. Phys. 1999, 111, 1343; b) V. Ledentu, W. Dong, P. Sautet, J. Am. Chem. Soc. 2000, 122, 1796.

[6] Thickness of PdC was calculated, assuming only inelastic scattering, using the formula of $\mathrm{d}=(\mathrm{IMFP} / \cos \Theta) *\left(1+\mathrm{I}^{335.6 \mathrm{eV}} / \mathrm{I}^{335 \mathrm{eV}}\right)$, where $\mathrm{I}$ is the corresponding peak area, $\Theta$ the takeoff angle, and IMFP the inelastic mean free path. IMFP was $9 \AA$ at $720 \mathrm{eV}$. The growth of PdC was determined from the temporal evolution of the thickness. excitation, corresponding to an inelastic mean free path of approximately $9 \AA .^{[15]}$

In situ Prompt Gamma Activation Analysis (PGAA) was carried out at the cold neutron beam of the Budapest Neutron Centre, Budapest, Hungary. ${ }^{[16]} \mathrm{H} / \mathrm{Pd}$ molar ratios of $7 \mathrm{mg}$ Pd black were determined under different conditions from the characteristic peak areas corrected by the detector efficiency and the nuclear data of the observed elements. $^{[8]}$

The 20 bar acetylene hydrogenation was carried out in a continuous-flow microreactor with on-line GC using a $0.02 \% \mathrm{Pd}$ /alumina catalyst.

Density Functional Theory based calculations on slab models have been carried out to investigate the accumulation of carbon on/in $\operatorname{Pd}(111)$. The adsorption energy was evaluated using the PW91 functional ${ }^{[17]}$. The PAW method ${ }^{[18]}$ was used to represent the inner cores and one electron states were expanded in a plane wave basis with a kinetic cut off energy of $400 \mathrm{eV}$. A Monkhorst-Pack mesh with $11 \times 11 \times 1$ and $9 \times 9 \times 1 \mathrm{k}$-points was used for small and large cells. ${ }^{[19]}$
[7] H. Yokoyama, H. Numakura, M. Koiwa, Acta mater. 1998, 46, 2823.

[8] Handbook of Prompt Gamma Activation Analysis with Neutron Beams, (Ed.: G. L. Molnár) Kluwer Academic Publishers, Dordrecht/London/Boston, 2004.

[9] Zs. Révay, T. Belgya, L. Szentmiklósi, Z. Kis, A. Wootsch, D. Teschner, M. Swoboda, R. Schlögl, J. Borsodi, R. Zepernick, Anal. Chem. DOI: 10.1021/ac800882k

[10] H. Frieske, E. Wicke, Ber. Bunsenges. 1973, 77, 48.

[11] G. Kresse, J. Hafner, Phys. Rev. B 1993, 47, 558.

[12] L. Gracia, M. Calatayud, J. Andrés, C. Minot, M. Salmeron, Phys. Rev. B 2005, 71, 033407.

[13] K. Reuter, M. Scheffler, Phys. Rev. B 2003, 68, 045407.

[14] a) W. Dong, V. Ledentu, P. Sautet, A. Eichler, J. Hafner, Surf. Sci. 1998, 411, 123. b) J.-F. Paul, P. Sautet, Phys. Rev. B 1996, 53, 8015 .

[15] S. Tanuma, C. J. Powell, D. R. Penn, Surf. Interface Anal. 1991, 17, 911.

[16] Zs. Révay, T. Belgya, Zs. Kasztovszky, J. L. Weil, G. L. Molnár, Nucl. Instrum. and Meth. B 2004, 213, 385.

[17] J. P. Perdew, J.A. Chevary, S. H. Vosko, K.A. Jackson, M. R. Pederson, D. J. Singh, C. Fiolhais, Phys. Rev. B 1992, 46, 6671.

[18] P. E. Blöchl, Phys. Rev. B 1994, 50, 17953.

[19] H. J. Monkhorst, J. D. Pack, Phys. Rev. B 1976, 13, 5188. 